\title{
Astaxanthin attenuates neurotoxicity in a mouse model of Parkinson's disease
}

\author{
Grimmig B., ${ }^{1}$ Daly. L., ${ }^{2}$ Hudson C., ${ }^{1,2}$ Nash KR., ${ }^{3}$ Bickford PC ${ }^{*, 4}$
}

${ }^{1}$ Department of Molecular Pharmacology and Physiology, USF Morsani College of Medicine, Tampa FL 33620, USA; ${ }^{2}$ Center of Excellence for Aging and Brain Repair, Department of Neurosurgery and Brain Repair, USF Morsani College of Medicine. Tampa, FL 33620, USA; ${ }^{3}$ Byrd Alzheimer's Institute, Department of Molecular Pharmacology and Physiology, Morsani College of Medicine, University of South Florida, Tampa, FL 33620, USA; ${ }^{4}$ Research Service, James A Haley Veterans Affairs Hospital, Tampa, FL 33620, USA

*Corresponding author: Paula C. Bickford, PhD, Center of Excellence for Aging and Brain Repair, MDC78, Department of Neurosurgery and Brain Repair, University of South Florida, College of Medicine, Tampa, FL, USA 33612, USA

Submission Date: March 22 ${ }^{\text {nd }}, 2017$, Acceptance Date: August 21 ${ }^{\text {st }}, 2017$, Publication Date: August $31^{\text {st }}, 2017$

Citation: Grimmig B., Daly. L., Hudson C., Nash KR., ${ }^{3}$ Bickford PC. Astaxanthin attenuates neurotoxicity in a mouse model of Parkinson's disease. Functional Foods in Health and Disease 2017; 7(8): 562-576. https://doi.org/10.31989/ffhd.v7i8.352

\begin{abstract}
Background: Astaxanthin (AXT) is a natural carotenoid with diverse biological activities. Although it is best known as a potent antioxidant, recent work suggests additional mechanisms of action that have the potential to oppose the ongoing pathophysiology of Parkinson's disease (PD). For example, AXT has a putative role in modulating microglial activity and preserving mitochondrial function, thereby implicating this compound as a neuroprotective agent. Both oxidative stress and inflammation are involved in the progression of many neurodegenerative diseases. Therefore, we examined the efficacy for AXT to reduced neurotoxicity in a toxic model of PD in mice.
\end{abstract}

Methods: In this study, we used a 4-week dietary supplementation of algae derived AXT to reduce 1-methyl-4-phenyl-1,2,3,6-tetrahydropyridine (MPTP) induced dopaminergic cell death.

Results: AXT treated mice were protected against the loss of tyrosine hydroxylase (TH) staining in the substantia nigra (SN) after MPTP exposure compared to the control diet. This effect of preserved TH immunoreactivity was also observed in the striatum. Furthermore, AXT administration was able to interrupt the neuroinflammatory process known to contribute to neurodegeneration in this model. 
Conclusion: We demonstrate that AXT neuroprotection was associated with attenuated microglial activation as indicated by reduced immunohistochemical detection of IBA-1 in the SN and striatum of AXT treated mice. Altogether, these studies suggest that AXT has neuroprotective property in the central nervous system against MPTP neurodegeneration.

Keywords: Astaxanthin, Neuroprotection, Neurodegeneration, Neuroinflammation, Parkinson's disease

\section{BACKGROUND}

Parkinson's disease (PD) is the second most common neurodegenerative disorder. PD is anatomically defined by the loss of dopaminergic innervation to the striatum, leading to the characteristic motor dysfunctions: bradykinesia, rigidity of the limbs and a shuffling gait. According to the Parkinson's Disease Foundation, PD is estimated to afflict 10 million people worldwide, and is thereby associated with a significant economic burden. Furthermore, PD is projected to become more prevalent as the largest sector of the US population gets older [1]. Currently, the commonly prescribed medications are based on dopamine replacement strategies. While they effectively mitigate motor symptoms temporarily, these drugs often become less effective with chronic use. The current treatment options are not adequate; there are still no medications available to stop or delay the progression of the disease. Therefore, there is still a pressing need to identify compounds capable of modulating the disease process and preventing the development of symptoms among these patients.

While the exact pathological mechanisms that initiate neurodegeneration in PD are still being investigated, it is accepted that multiple biological processes are impacted sometime during the course of the disease that likely interact with and perpetuate the pathogenesis. Both neuroinflammation and oxidative stress are thought to be involved in neurotoxic cascades and contribute to cell loss. As a result, there has been significant focus on developing therapeutic strategies to attenuate these aberrant conditions in an attempt to alleviate some of the cellular stress that causes neuronal dysfunction.

Astaxanthin (AXT) is a xanthophyll carotenoid produced primarily by the marine algae Heamatococcus Pluvialis. AXT is currently available as a health supplement, being marketed for its antioxidant capacity; however, recent research indicates that AXT has multiple putative mechanisms of action responsible for its various health benefits [2]. This compound is being investigated in relation to multiple clinical conditions including cardiovascular disease, metabolic syndrome, and athletic performance [3-5]. Interestingly, emerging evidence suggests that the proposed biological activities of AXT precisely oppose the pathophysiology that underlies Parkinson's disease, revealing a distinct and promising therapeutic potential in the prevention or delayed onset of symptoms in PD patients.

AXT is best known for its potent antioxidant activity, reported to be much more effective than other similar compounds [6]. This is likely due to the numerous actions as an antioxidant, as it can reduce radicals by absorption, donation of electrons, and formations of adducts with the reactive species. The presence of hydroxylated ionone rings that cap both ends of the carbon backbone distinguish the molecule from other carotenoids in its class, and enables AXT energetically 
favorable spanning the phospholipid bilayer of cell membranes. This orientation and chemical structure effectively protects the membrane against lipid peroxidation [3].

Many reports indicate that AXT reduces the evidence of oxidative damage [7-11]. AXT can also stimulate the expression or the activity of endogenous antioxidant enzymes, including glutathione [7, 12-14]. Glutathione is expressed in low levels in the substantia nigra, being one of the factors that renders the SN more susceptible to oxidative damage compared to adjacent brain structures [15]. It has also been shown that there is less reduced glutathione in the brains of PD patients, which further indicates the involvement of this molecule and the oxidative status of the SN to clinical presentation [16]. Elevated levels of reactive oxygen species in this region promote pathological modifications of $\alpha$-synuclein. For example, nitrosylated $\alpha$-synuclein that readily occurs in an oxidized environment has been shown to stimulate microglial output of proinflammatory compounds and is thereby associated with neuronal dysfunction and degeneration [17]. AXT may lower oxidative stress by directly neutralizing radicals and also through the modulation of antioxidant response signaling cascades.

AXT has also been suggested to modulate the immune response to various insults, which indicates that there is a potential anti-inflammatory action for this compound. Multiple investigators have demonstrated that AXT can reduce the expression of inflammatory mediators including: inducible nitric oxide synthase and nitric oxide (iNOS/NO), nuclear factor kappa-lightchain-enhancer of activated B cells (NFkB), and interleukin 6 (IL-6) [11, 18-20]. These secreted factors are released in high amounts by activated microglia and are associated with neuronal damage. A modulatory action of AXT on these microglial responses to brain insults represents a possible approach to interrupting one of the factors known to perpetuate neuronal dysfunction in the PD brain.

Interestingly, AXT has been shown to be protective in cell culture systems against the toxic compounds used to produce in vitro experimental models of PD. For example, Lui et al. (2009) demonstrate that AXT pretreatment effectively protects SHSY5Y cells against 6hydroxydopamine, a commonly used toxin that degenerates the dopaminergic cells primarily as an oxidative insult. In this study, AXT attenuated neurotoxicity was associated with reduced ROS production, in addition to DNA fragmentation, cleavage of poly (ADP-ribose) polymerase, and cytosolic cytochrome C [9]. AXT pretreatments have also successfully protected PC 12 cells from 1-methyl-4-phenylpyridinium (MPP+) [14, 21-23]. MPP+ is a toxic derivative of 1-methyl-4phenyl-1,2,3,6-tetrahydropyridine (MPTP), another established agent used to recapitulate dopaminergic cell degeneration and is also widely used to model PD. In light of how AXT has been shown to be protective in cell culture systems against PD specific compounds, we evaluated the efficacy of AXT supplementation to prevent neurodegeneration in vivo at a physiological relevant dose.

In the present study, we explore the capacity for a dietary pre-treatment of AXT to protect against neuronal damage caused by the neurotoxin MPTP. C57BL6 mice (3 months) were treated with AXT for one month. These mice consumed an AXT enriched diet formulated to deliver a dose of $3 \mathrm{mg} / \mathrm{kg} /$ day (treatment group-AXT). Multiple studies have reported a range of doses of AXT that were used therapeutically across various disease models; this dose was chosen in order to determine the efficacy of the physiological levels that would occur in humans taking the recommended dose of commercially available dietary supplements. Additional groups were fed 
the standard grain based rodent diet (control treatment groups; CTL) or the control diet containing the inactive ingredients of the AXT supplement product to serve as the vehicle control condition (vehicle treatment group; VEH). After the month of dietary pre-treatment, half of the mice from each dietary condition received 4 injections of MPTP at $10 \mathrm{mg} / \mathrm{kg}$ intraperitoneal once per hour for 4 hours to achieve a total dose of $40 \mathrm{mg} / \mathrm{kg}$. They were then allowed to recover for 7 days before euthanasia. In this study, we show that the AXT supplemented diet protected against neurodegeneration and inflammation which typically occurs after exposure to MPTP.

\section{METHODS}

All procedures were conducted according to the National Institute of Health Guide and Use of Laboratory Animals and the University of South Florida IACUC. Male C57BL/6J mice at 3 months of age were maintained in environmentally controlled conditions of $21^{\circ} \mathrm{C}$ under a 12 hour light/dark cycle. The mice were allowed ad libitum access to food and water. However, the food was consistently monitored and replaced daily by research staff to maintain freshness.

Dietary pretreatment: Mice were treated with $3 \mathrm{mg} / \mathrm{kg}$ of Bioastin ${ }^{\circledR}$ generously supplied by Cyanotech. This is a natural astaxanthin product delivered on inert cellulose beads with trace amounts of vitamin E. For this reason, we included a separate group treated with the empty beads to serve as a vehicle control. These compounds were incorporated into the Harlan Teklad rodent diet, and delivered ad libitum for 1 month prior to MPTP administration. Food intake per cage and individual body weights were monitored throughout the course of treatment to ensure consumption.

MPTP administration: The MPTP-HCL (Sigma-Aldrich) was diluted in sterile saline and injected intraperitoneally. The mice received four injections at a dose of $10 \mathrm{mg} / \mathrm{kg}$, administered once per hour four hours for a final dose of $40 \mathrm{mg} / \mathrm{kg}$ MPTP or equal volumes of sterile saline for control conditions. Mice were allowed to recover from MPTP exposure for 7 days before they were fully anesthetized with phenobarbital and transcardially perfused with phosphate buffered saline. Brains were immediately removed and one hemisphere was microdissected for areas of interest. The other hemisphere was preserved in $4 \%$ paraformaldehyde for 24 hours. The brains were then transferred to a $30 \%$ sucrose solution for cryoprotection.

Immunohistochemistry: $40 \mu \mathrm{m}$ coronal sections were selected at a periodicity of 1 in 6 for all immunohistochemical procedures; additional sections were included both anterior and posterior to the $\mathrm{SN}$ to ensure thorough representation of the area of interest. Free floating sections were incubated in primary antibody (TH: Immunostar 1:1,000, IBA1: Wako Laboratory Chemicals 1:2,500, NeuN: Millipore 1:5,000) diluted in goat serum and TX-100 for 24 hours at $4^{\circ} \mathrm{C}$ and secondary antibody for 60 minutes at $25^{\circ}$. After incubation in avidin-biotin complex (Vector Labs), precipitation reactions were developed with diaminobenzidine (Sigma-Aldrich).

Quantification: Immunoreactivity was quantified using an AxioScan microscope (20X objective) and NearCYTE image analysis software (nearcyte.org). This program applies a user defined 
threshold of color intensity to images of the sections and generates a ratio of positive staining within a region of interest. Our lab has previously determined that data collected with the NearCYTE software accurately reflects stereological cell counts [24].

Glutathione assay: Briefly, plasma samples isolated from whole blood were immediately diluted 1:20 in a 5\% solution of sulfosalicylic acid for deproteination and preservation. All subsequent steps in this assay was performed according to manufacturer's instructions (Arbor Assays).

GDNF ELISA: Briefly, snap frozen samples of striatum were quickly homogenized and centrifuged to collect lysates. Protein concentration was determined and $40 \mathrm{ug}$ of sample was loaded into the wells while the rest of the assay was conducted according to manufacturer's instructions (BOSTERbio).

Data Analysis: The data presented graphically as the group mean and standard error of the mean. Statistical analysis was performed using GraphPad Prism software. 2 Way ANOVAs were conducted, while Bonferroni multiple comparisons posthoc tests (unless otherwise specified) were used to further compare differences between groups.

\section{RESULTS}

\section{AXT prevents MPTP induced neurodegeneration}

As expected, we observed approximately $50 \%$ loss of tyrosine hydroxylase positive staining in the substantia nigra of control mice one week after MPTP administration. Mice that were fed a vehicle control diet demonstrated a similar TH loss as the controls. However, the AXT enriched diet was able to preserve the TH expression in this region (Figure 1).
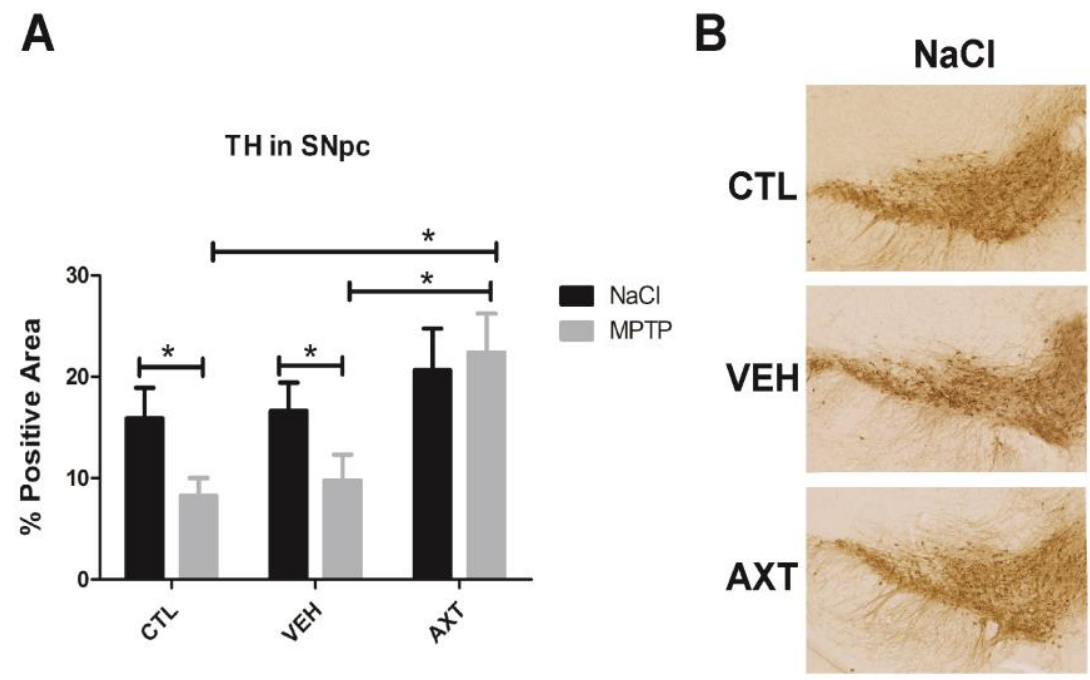

\section{MPTP}

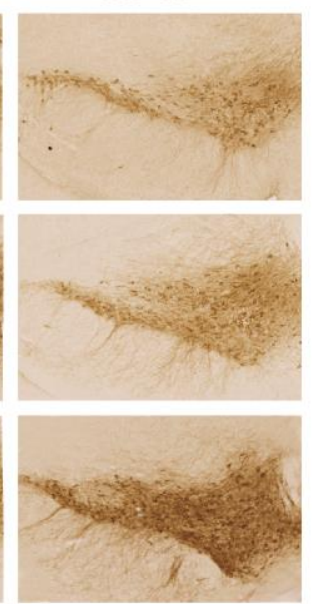

Figure 1. One month dietary supplementation with AXT preserves $\mathrm{TH}$ immunoreactivity in the substantia nigra pars compacta in response to MPTP. A) Bar graph of percent area of positive staining of TH relative to a user defined threshold. B) Immunohistochemistry with anti-TH antibody in the SN. 2-way ANOVA; diet effect: DF 2, F 4.458; Bonferroni posthoc p<0.05; Scale $=100 \mu \mathrm{m}$ 
Likewise, we also observed reduced positive staining for tyrosine hydroxylase in the striatum of mice that were fed the control and vehicle diets. However, mice that consumed a diet enriched with AXT were protected from this loss of TH immunoreactivity, indicating less destruction of the terminals that innervate the basal ganglia (Figure 2). It is known that MPTP treatment can directly reduce the levels and efficacy of the TH enzyme, and this deficit may be transient. Therefore, an additional immunohistochemical assessment was conducted to assess the surviving neurons in the $\mathrm{SN}$. The digital image quantification of neuronal nuclei $(\mathrm{NeuN})$ staining revealed that the AXT enriched diet reduced neuron loss 7 days after MPTP exposure in the SN compared to the other diets, corroborating the trends observed in TH retention (Figure 3).

A

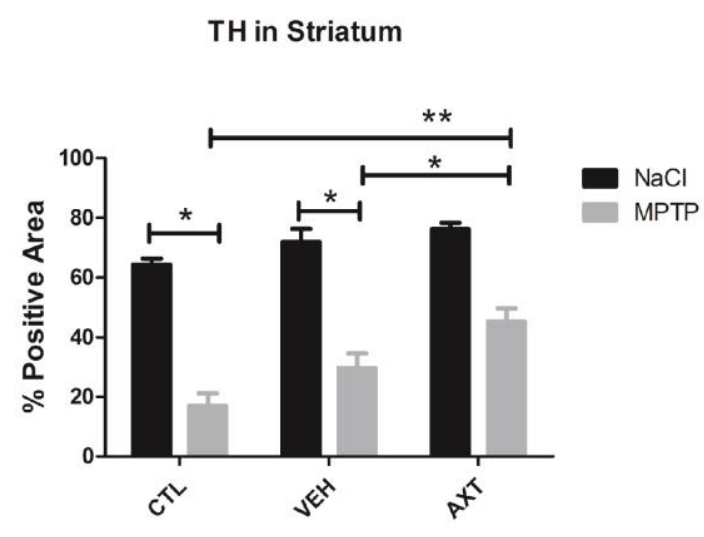

B

CTL

VEH

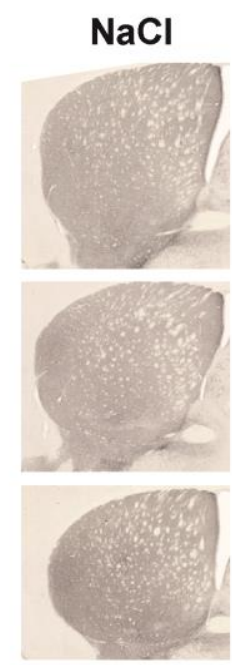

MPTP

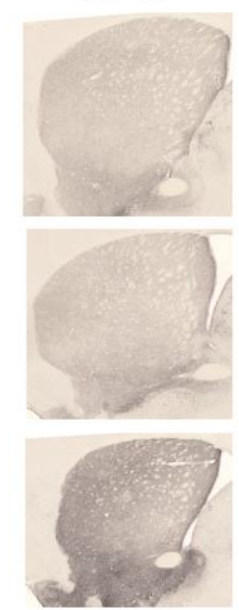

Figure 2. One month dietary supplementation with AXT reduces TH immunoreactivity loss in the striatum in response to MPTP. A) Bar graph of percent area of positive staining of TH relative to a user defined threshold. B) Immunohistochemistry with anti-TH antibody in the striatum. 2 way ANOVA, Diet effect DF 2, F 14.08 Newman-Keuls *p $<0.05 * * \mathrm{p}<0.001$; Scale $=200 \mu \mathrm{m}$

A

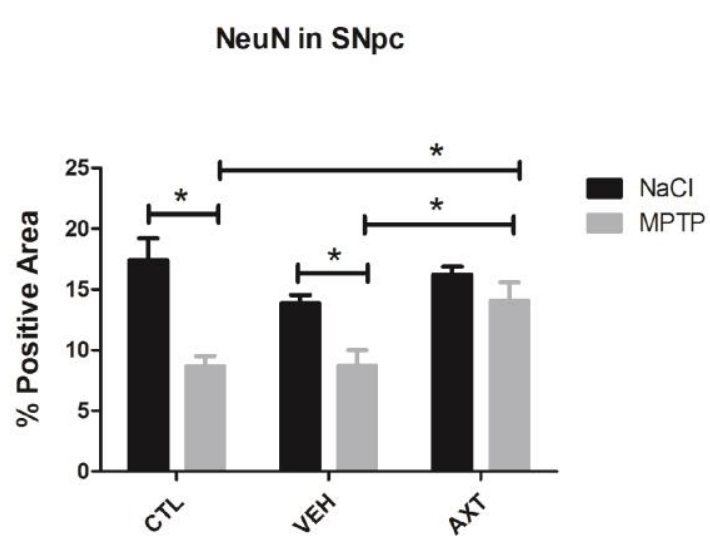

B

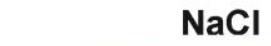

CTL

VEH

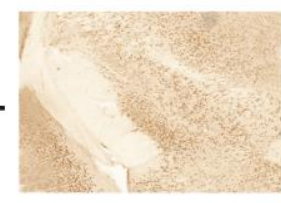

AXT
MPTP

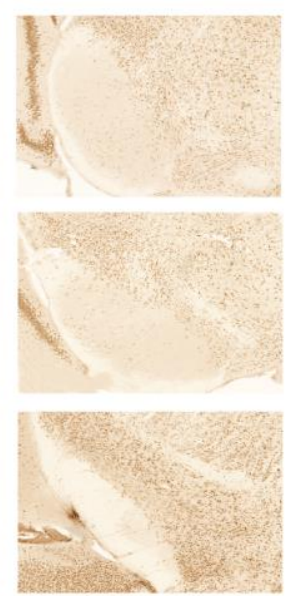

Figure 3. One month dietary supplementation with AXT preserves neurons in the $\mathrm{SN}$ in response to MPTP. A) Bar graph of percent area of positive staining of NeuN relative to a user defined threshold. B) Immunohistochemistry with anti-NeuN antibody in the SN. 2-way ANOVA, Diet effect DF 2, F 4.2. Tukey multiple comparison $\mathrm{p}<0.05$; Scale $=200 \mu \mathrm{m}$ 


\section{AXT alters microglial response to MPTP neurotoxicity}

It has been postulated that AXT may modulate microglial function and their reactivity to various insults $[18,25,26]$. Microglial express ionized calcium-binding adaptor molecule protein 1 (IBA1) at basal levels, but are known to upregulate this molecule when exposed to certain immune stimuli; the induction of IBA1 typically indicates microglial activation. Therefore, we examined the expression of IBA1 in the striatum and SN. In this study, we demonstrate how MPTP leads to an increase of IBA1 in the striatum in the control and approaching significance in VEH treated diets. In contrast, AXT supplementation minimized the increase in expression of IBA1 (Figure 4). Interestingly, we did not observe a significant increase in IBA1 in the SN of control MPTP treated animals but we observed that AXT treatment reduced basal levels of microglial expression of IBA1 in the SN compared to control mice (Figure 5). This lower basal level of IBA1 was maintained in the MPTP treated group with AXT treatment (Figure 5).

A

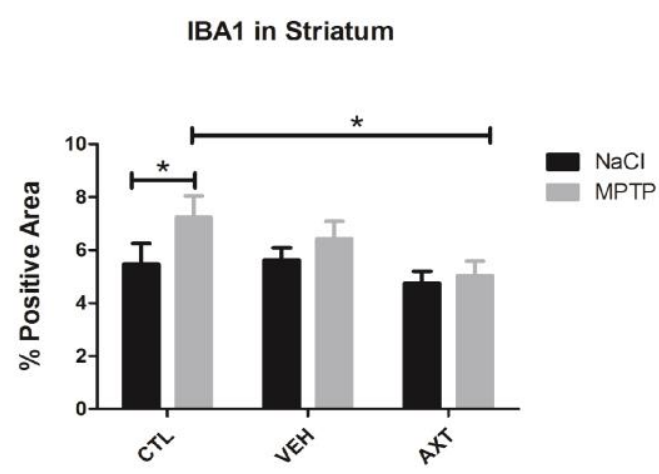

B

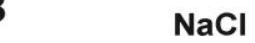

CTL

VEH

AXT
$\mathrm{NaCl}$

MPTP

Figure 4. Dietary intervention of AXT modulates microglial response to the neurodegeneration that results from MPTP neurotoxicity. A) Bar graph of percent area with positive staining of IBA1 in the striatum relative to a user defined threshold. MPTP induced upregulation of the microglial marker IBA1 is attenuated in the striatum of mice being treated with AXT 2-way ANOVA, Diet effect DF 2, $3.3 \mathrm{~F}$; Tukey's multiple comparison $\left.\left({ }^{*} \mathrm{p}<0.05\right) ; \mathrm{B}\right)$ Immunohistochemistry with anti-IBA1 antibody in the striatum. Scale $=20 \mu \mathrm{m}$

A

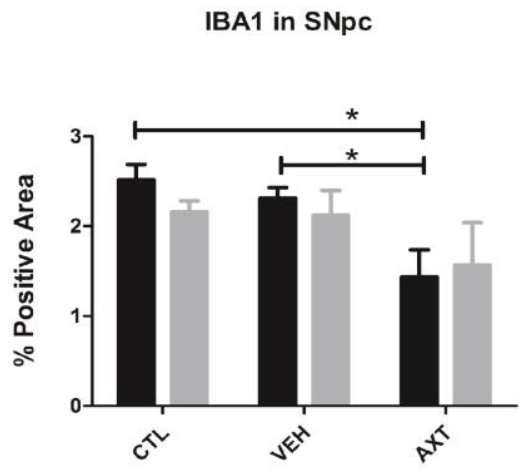

B

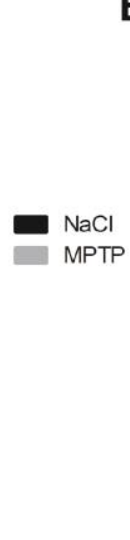

$\mathrm{NaCl}$

CTL

VEH

AXT
MPTP

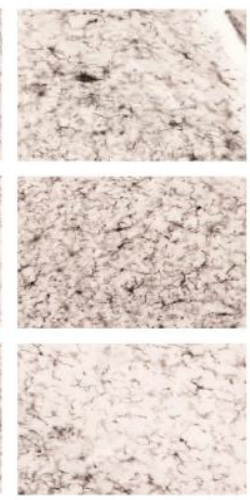

Figure 5. Dietary intervention of AXT modulates microglial response in the SN. A) Bar graph of percent area with positive staining of IBA1 relative to a user defined threshold. IBA1 in the SN is reduced with AXT treatment. 1 way ANOVA of saline treatment with Bonferroni multiple comparison $\left({ }^{*} \mathrm{p}<0.05\right)$; B) Immunohistochemistry with anti-IBA1 antibody in the SN. Scale $=20 \mu \mathrm{m}$ 


\section{Antioxidant effect}

Oxidative stress is considered to be involved in both the pathogenesis of PD and to contribute to the neurotoxicity of MPTP [27]. Many therapeutic strategies aim to mitigate the damage caused by excessive release of reactive oxygen species. It has been suggested that AXT can increase the efficacy of endogenous antioxidant enzymes, either by increasing their expression levels or their enzymatic activity. As previously mentioned, it is well documented that AXT has an antioxidant effect and can protect biological systems, including both cell culture and whole organisms from oxidative damage, $[10,11,14,23,28]$. Therefore, we assessed the levels of glutathione in the plasma. In our study, one month of AXT supplementation significantly elevated the ratio of reduced glutathione (GSH) to oxidized glutathione (GSSH). Even after exposure to MPTP, the AXT treated animals still retained a more favorable ratio of GSH to GSSH than the control mice, despite the increased output of reactive oxygen species known to occur in this model (Figure 6). In contrast, mice on the standard control diet had lower levels of GSH after MPTP exposure.

A

Glutathione in Plasma

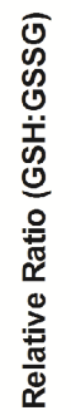
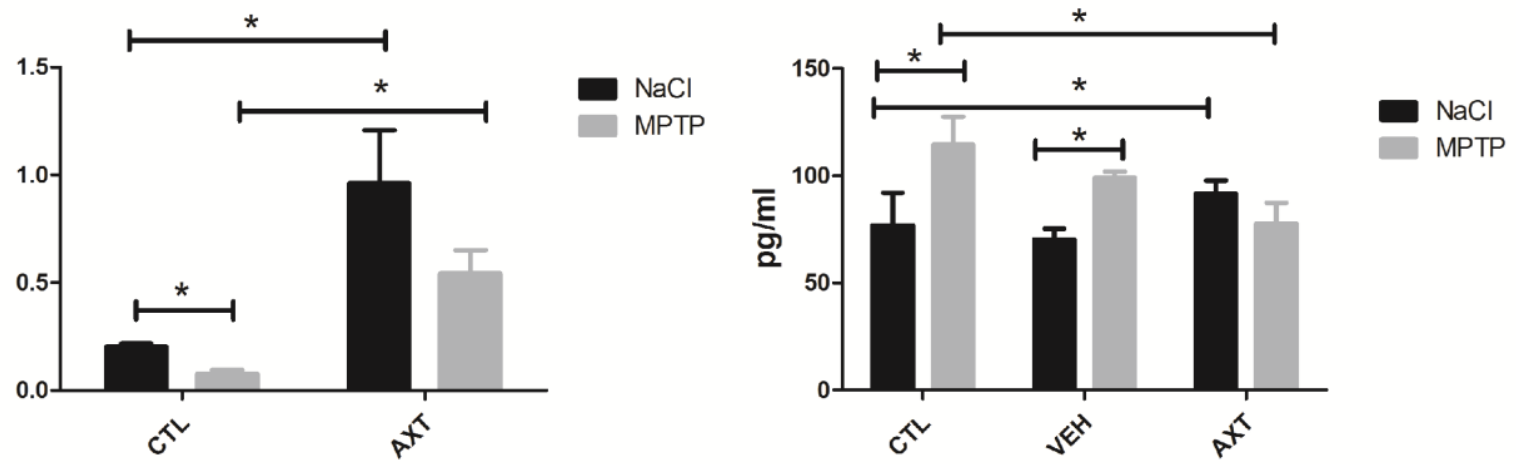

Figure 6. A) AXT increases the ratio of reduced glutathione (GSH) to oxidized glutathione (GSSG). Bar graph of the relative ratio of GSH:GSSH in plasma. AXT increases the ratio in both MPTP treated and non-MPTP animals compared to control animals. 2-way ANOVA Diet effect: DF 1, F 14.51; Bonferroni multiple comparisons $\left({ }^{*} \mathrm{p}<0.05\right)$. B) MPTP exposure is associated with increased expression of GDNF in the striatum. AXT treatment suppresses the elevation of GDNF in response to MPTP. 2 way ANOVA Diet effect: DF 1, F 3.8: Bonferroni multiple comparisons $\left({ }^{*} \mathrm{p}<0.05\right)$ and independent T-tests.

\section{AXT modulates growth factor expression in the striatum}

It has been shown that AXT can stimulate the expression of brain derived neurotrophic factors in various brain regions in vivo. We evaluated another growth factor, GDNF, because of its expression in the striatum and relevance to Parkinson's disease. GDNF is necessary for the postnatal development of dopaminergic cells within the midbrain and stimulates neurite outgrowth, facilitating integration and connectivity of the new cells. Incubating fetal grafts in GDNF also aids in the survival of transplantation into the ventral midbrain [29, 30]. GDNF has been evaluated for its therapeutic potential for supporting fiber growth and re-innervation of the striatum during the progressive cell loss in PD. Several preclinical animal studies have demonstrated that enhancing GDNF expression within the striatum can offer protection against toxic injury. For example, the viral vector driven expression of GDNF in the striatum of the 
common marmoset was found to be protective against the degeneration of dopaminergic terminals caused by 6-hydroxydopamine injections. This neuroprotection was observed with GDNF levels moderately above physiological levels and was associated with improved behavioral outcomes compared to the lesioned animals [31]. In this study, we show how MPTP treated mice on either the control or vehicle control diets have higher levels of GDNF in the striatum compared to the saline injected animals (Figure 6). In contrast, we did not observe this increase in the mice that consumed the AXT enriched diet. Furthermore, AXT treatment alone seemed to elevate the expression of GDNF in the striatum compared to the control diet in the saline injected mice.

\section{DISCUSSION}

While PD is common among the elderly, there are no available medications to cure, prevent, or delay the progression of the disease. Levidopa and other routinely prescribed medications are only palliative; they are effective at restoring dopamine. However, this only addresses motor symptoms and does not prevent ongoing neurodegeneration. Therefore, elucidating disease modifying agents and developing strategies to delay or prevent the onset of symptoms are critical for the treatment of PD. AXT is a possible disease modifying agent that can interrupt various mechanisms involved in the toxic cascade of MPTP. This is noteworthy because neurotoxic mechanisms of MPTP, such as oxidative damage, mitochondrial dysfunction (complex 1 impairment), and neuroinflammation are also known to be involved in the pathogenesis of PD. Other studies have supported the therapeutic strategy of using natural compounds in the treatment of disorders with similar pathological elements of inflammation and oxidative stress [32]. Mitigating multiple pathological mechanisms is a clear advantage of an AXT based therapeutic strategy. Furthermore, this compound can be considered for use chronically as a preventative or adjuvant therapy, as there are little to no adverse effects reported to date.

AXT is known to cross the blood brain barrier and accumulate within the brain tissue in addition to other organs [33]. Multiple studies have shown that AXT may also be beneficial specifically within the central nervous system for a variety of neurological injuries and disorders. For example, AXT supplementation has been purposed to augment cognitive function and neuroplasticity something which has been recently reviewed [34]. AXT was protective in a mouse model of traumatic brain injury [35]. Ji et al (2017) demonstrated that oral AXT treatment prevented the loss of cortical neurons and the associated behavioral impairments usually detectable in mice after traumatic brain injury. Similarly, AXT administration was also able to reduce the damage in a model of subarachnoid hemorrhage; AXT decreased edema, blood brain barrier dysfunction, and oxidative damage ultimately contributed to neuronal loss observed in the control animals [28]. These recent in vivo reports support the therapeutic potential of AXT for neurological based diseases.

In the present study, we used astaxanthin, a naturally derived carotenoid, to mitigate the neurotoxicity using an MPTP mouse model of Parkinson's disease. Our data suggests that the mechanisms underlying AXT mediated neuroprotection are related to modulating inflammation and oxidative stress. We determined that AXT supplementation effectively protects against the loss of dopaminergic neurons in the substantia nigra and their TH enriched terminals in the striatum. We also show that dietary AXT administration altered the microglial response to MPTP 
induced neurodegeneration, as IBA1 induction was not observed in the AXT treated animals. These results are consistent with previous work showing how AXT in cell culture can attenuate the toxicity of 6-hydroxydopamine and MPP+ [9, 14, 21, 23]. It also corroborates many preliminary studies that demonstrate AXT treatment may modulate the output of inflammatory cytokines. For example, AXT has been shown to reduce transcripts of proinflammatory mediators [20]. Functionally, the administration of AXT can block some inflammatory sequelae of the immunogen lipopolysaccharide injections [25] and can inhibit NFkB translocation to the nucleus, thereby down-regulating TNF- $\alpha$ expression [36]. Treatments that reduce microglial activation such as fractalkine [37] or the Rho Kinase inhibitor Fasudil [38] are known to reduce the neurotoxicity of MPTP. Therefore, it is likely that the effect of AXT to reduce IBA-1 is directly related to the neuroprotection observed in this study.

We also show that AXT intake was associated with a more favorable ratio of GSH reduced glutathione to GSSH, oxidized glutathione in the plasma. Reduced glutathione indicates less oxidative stress, possibly either from increased levels or increased efficiency of glutathione to neutralize free radicals in the cellular environment. As discussed previously, glutathione status is of interest in Parkinson's disease, as the levels of this endogenous antioxidant molecule are substantially decreased within the PD brain and is thought to perpetuate oxidative damage that contributes to neuronal atrophy. Interestingly, pharmacological reduction of glutathione in vitro has been directly linked to nitrosylation of mitochondrial proteins leading to complex 1 deficiency. Mitochondrial dysfunction is another pathological characteristic of the PD brain [39]. Furthermore, measuring glutathione in the plasma correlates with the severity of cognitive dysfunction in Alzheimer's disease patients [40] confirming that there is a relationship between peripheral GSH levels and brain function.

The AXT modulation of glutathione observed in this experiment is consistent with other studies. Mattei et al. (2011) also demonstrates how AXT administration leads to improved antioxidant capacity including an increased ratio of GSH:GSSH in the plasma [41]. AXT was shown to increase GSH levels in the liver of animals exposed to the chemical carcinogen carbon tetrachloride $\left(\mathrm{CCl}_{4}\right)$. Kang et al. (2001) attributed the attenuation of $\mathrm{CCL}_{4}$ induced liver toxicity to the AXT stimulation of the endogenous antioxidant system [42]. Likewise, AXT has also been shown to reduce excitotoxicity in an animal model of epilepsy. These authors demonstrate that AXT treatment reduced neuronal apoptosis and upstream oxidative damage induced by overstimulation of the amygdala. This protective effect was associated with an increase in glutathione and a decrease output of reactive oxygen species [10]. While oxidative stress plays a role in the pathogenesis of Parkinson's disease, it is not the only major biological process negatively impacted during the course of disorder. It is notable that many antioxidant compounds have failed in clinical trials. While the antioxidant capacity of AXT likely reduces some neuronal dysfunction due to minimizing rampant oxidative damage, other suggested mechanisms of action likely come together and work in a synergistic or cumulative way to reduce the neurodegeneration seen in this model.

Finally, we observed an AXT mediated effect on GDNF expression in the striatum. As previously mentioned, GDNF has been investigated as a therapeutic target in the treatment of 
Parkinson's disease. Preclinical animal work supports the efficacy of GDNF based therapies as this growth factor has been shown to both be necessary for post-natal dopaminergic cell differentiation and survival in the mesencephalon, in addition to enhancing the successful integration of fetal tissue transplanted into the ventral midbrain [29, 30]. Additionally, upregulating GDNF expression above physiological levels using adeno associated viral vector effectively protected against degeneration caused by the 6-hydroxydopamine toxin and prevented the subsequent behavioral impairments [31]. It is known that growth factor expression can by stimulated by neuronal injury in effort to restore the damaged site [43]. We speculate that the increase of GDNF that we observed after MPTP exposure in our experiment was a compensatory mechanism in response to the lesion formed after the toxic insult. Likewise, it is also possible that even a subtle increase in striatal GDNF in the mice supplemented with AXT was sufficient to reduce some of the cell death caused by MPTP in the first place.

\section{CONCLUSION}

In conclusion, we have demonstrated an effect of AXT to reduce neurodegeneration in the MPTP model of PD. We show a concomitant change in the factors of inflammation and oxidative stress, suggesting these processes are associated and likely mediate the protective effect of AXT against MPTP. MPTP is a potent neurotoxin, and one caveat of this model is its rapid time course and dramatic dopaminergic cell loss does not recapitulate the slow progression of pathology seen in PD patients. Accordingly, future studies that use AXT in additional models of PD, like the viral vector mediated over expression of synuclein, will establish a role in disease progression as well as prevention.

List of Abbreviations: AXT, astaxanthin; MPP+, 1-methyl-4-phenylpyridinium; MPTP, 1methyl-4-phenyl-1,2,3,6-tetrahydropyridine; SN substantia nigra; STR, striatum; iNOS/NO, inducible nitric oxide synthase and nitric oxide; NFкB, nuclear factor kappa-light-chain-enhancer of activated B cells; IL-6, interleukin 6; TH tyrosine hydroxylase; NeuN, neuronal nuclei; IBA1, ionized calcium-binding adaptor molecule protein 1; GSH, reduced glutathione; GSSH, oxidized glutathione; GDNF, glial derived neurotrophic factor; $\mathrm{CCl}_{4}$, carcinogen carbon tetrachloride.

Competing Interests: PCB serves on the scientific advisory board for Nutrex, Hawaii, the company that provided the AXT.

Authors' Contributions: BG, $\mathrm{CH}$, and LD conducted experiments, processed tissues, collected and analyzed data. $\mathrm{BG}, \mathrm{KN}$, and PCB designed experiments and interpreted data. BG, $\mathrm{LD}, \mathrm{KN}$, and PCB contributed to writing the manuscript.

Acknowledgments and Funding: We thank Cyanotech for generously supplying the Bioastin. Grant support: VA MRS grants I01BX003421; I01BX000231 (PCB); NIH R01AG044919 (PCB). This work was supported by the Veterans administration. However, the content of this manuscript does not reflect the views and opinions of the Veterans Administration or the US government. 


\section{REFERENCES}

1. Kowal SL, Dall RTM, Chakrabarti MVS, Jain A: The current and projected economic burden of Parkinson's disease in the United States. Movement Disorders 2013, 28(3): 311-318.

2. Yamashita E: Astaxanthin as a Medical Food Functional Foods in Health and Disease 2013, 3(7): 254-258.

3. Kidd P: Astaxanthin, cell membrane nutrient with diverse clinical benefits and antiaging potential. Altern Med Rev 2011, 16(4): 355-64.

4. Hussein G, Nakagawa T, Goto H, Shimada Y, Matsumoto K, Sanktawa U, Watanabe $\mathrm{H}$ : Astaxanthin ameliorates features of metabolic syndrome in SHR/NDmcr-cp. Life Sci 2007, 80(6): 522-9.

5. Djordjevic B, Baralic I, Kotur-Stevuljevic J, Stefanovic A, Ivanisevic J, Radivojevic N, Andjelkovic M, Dikic N: Effect of astaxanthin supplementation on muscle damage and oxidative stress markers in elite young soccer players. J Sports Med Phys Fitness 2012, 52(4): 382-92.

6. Ambati RR, Siew Moi P, Ravi S, Aswathanarayana RG: Astaxanthin: Sources, Extraction, Stability, Biological Activities and Its Commercial Applications-A Review. Marine Drugs 2014, 12(1): 128-152.

7. Al-Amin MM, Akhter S, Hasan AT, Alam T, Nageeb Hasan SM, Saifullah AR, Shohel M: The antioxidant effect of astaxanthin is higher in young mice than aged: a region specific study on brain. Metab Brain Dis 2015, 30(5): 1237-46.

8. Guerin M, ME Huntley, M Olaizola: Haematococcus astaxanthin: applications for human health and nutrition. Trends Biotechnol 2003, 21(5): 210-6.

9. Liu X, Shibata T, Hisaka S, Osawa T: Astaxanthin inhibits reactive oxygen speciesmediated cellular toxicity in dopaminergic SH-SY5Y cells via mitochondria-targeted protective mechanism. Brain Res 2009, 1254: 18-27.

10. Lu Y, Xie T, He XX, Mao ZF, Jia LJ, Wang WP, Zhen JL, Liu LM: Astaxanthin rescues neuron loss and attenuates oxidative stress induced by amygdala kindling in adult rat hippocampus. Neuroscience Letters 2015, 597: 49-53.

11. Park JS, Mathison BD, Hayek MG, Zhang J, Reinhart GA, Chew BP: Astaxanthin modulates age-associated mitochondrial dysfunction in healthy dogs. J Anim Sci 2013, 91(1): 268-75.

12. Saw CL, AY, Yang Y, Guo, Kong AN: Astaxanthin and omega-3 fatty acids individually and in combination protect against oxidative stress via the Nrf2-ARE pathway. Food Chem Toxicol 2013, 62: 869-75.

13. Wu Q, Zhang XS, Wang HD, Zhang X, Yu Q, Li W, Zhou ML, Wang XL, Astaxanthin activates nuclear factor erythroid-related factor 2 and the antioxidant responsive element (Nrf2-ARE) pathway in the brain after subarachnoid hemorrhage in rats and attenuates early brain injury. Mar Drugs 2014, 12(12): 6125-41. 
14. Ye Q, Huang B, Zhang X, Zhu Y, Chen X: Astaxanthin protects against MPP(+)induced oxidative stress in PC12 cells via the HO-1/NOX2 axis. BMC Neurosci 2012, 13: 156.

15. Sian J, Dexter DT, Lees AJ, Daniel S, Agid Y, Javoy-Agid F, Jenner P, Marsden CD: Alterations in glutathione levels in Parkinson's disease and other neurodegenerative disorders affecting basal ganglia. Ann Neurol 1994, 36(3): 348-55.

16. Sofic E, Lange KW, Jellinger K, Riederer P: Reduced and oxidized glutathione in the substantia nigra of patients with Parkinson's disease. Neurosci Lett 1992, 142(2): 12830.

17. Reynolds AD, Glanzer JG, Kadiu I, Ricardo-Dukelow M, Chaudhuri A, Ciborowski P, Cerny R, Gelman B, Thomas MP, Mosley RL, Gendelman HE: Nitrated alphasynuclein-activated microglial profiling for Parkinson's disease. J Neurochem 2008, 104(6): 1504-25.

18. Kim YH, Koh HK, Kim DS: Down-regulation of IL-6 production by astaxanthin via ERK-, MSK-, and NF-kappaB-mediated signals in activated microglia. Int Immunopharmacol 2010, 10(12): 1560-72.

19. Balietti M, Giannubilo SR, Giorgetti B, Solazzi M, Turi A, Casoli T, Ciavattini A, Fattorettia P: The effect of astaxanthin on the aging rat brain: gender-related differences in modulating inflammation. J Sci Food Agric 2016 96(2): 615-8.

20. Yook JS, Okamoto M, Rakwal R, Shibato J, Lee MC, Matsui T, Chang H, Cho JY, Soya $\mathrm{H}$ : Astaxanthin supplementation enhances adult hippocampal neurogenesis and spatial memory in mice. Mol Nutr Food Res 2016, 60(3): 589-99.

21. Chan KC, Mong MC, Yin MC: Antioxidative and anti-inflammatory neuroprotective effects of astaxanthin and canthaxanthin in nerve growth factor differentiated PC12 cells. J Food Sci 2009, 74(7): H225-31.

22. Lee DH, Kim CS, Lee YJ: Astaxanthin protects against MPTP/MPP+-induced mitochondrial dysfunction and ROS production in vivo and in vitro. Food Chem Toxicol 2011, 49(1): 271-80.

23. Ye Q, Zhang X, Huang B, Zhu Y, Chen X: Astaxanthin suppresses MPP(+)-induced oxidative damage in PC12 cells through a Sp1/NR1 signaling pathway. Mar Drugs, 2013, 11(4): 1019-34.

24. Nash KR, Moran P, Finneran DJ, Hudson C, Robinson J, Morgan D, Bickford PC: Fractalkine over expression suppresses alpha-synuclein-mediated neurodegeneration. Mol Ther 2015, 23(1): 17-23.

25. Al-Amin MM, Sultana R, Sultana S, Rahman MM, Reza HM: Astaxanthin ameliorates prenatal LPS-exposed behavioral deficits and oxidative stress in adult offspring. BMC Neurosci 2016, 17: 11.

26. Choi SK, Park YS, Choi DK, Chang HI: Effects of astaxanthin on the production of NO and the expression of COX-2 and iNOS in LPS-stimulated BV2 microglial cells. J Microbiol Biotechnol 2008, 18(12): 1990-6. 
27. Blesa J, Trigo-Damas I, Quiroga-Varela A, Jackson-Lewis VR: Oxidative stress and Parkinson's disease. Frontiers in Neuroanatomy 2015, 9: p91.

28. Zhang XS, Zhang X, Zhou ML, Zhou XM, Li N, Li W, Cong ZX, Sun Q, Zhuang Z, Wang CX, Shi JX: Amelioration of oxidative stress and protection against early brain injury by astaxanthin after experimental subarachnoid hemorrhage. J Neurosurg 2014, 121(1): 42-54.

29. Granholm AC, Reyland M, Albeck D, Sanders L, Gerhardt G, Hoernig G, Shen L, Westphal H, Hoffer B: Glial Cell Line-Derived Neurotrophic Factor Is Essential for Postnatal Survival of Midbrain Dopamine Neurons. The Journal of Neuroscience 2000, 20(9): 3182.

30. Granholm AC, Mott JL, Bowenkamp K, Eken S, Henry S, Hoffer BJ, Lapchak PA, Palmer MR, van Horne C, Gerhardt GA: Glial cell line-derived neurotrophic factor improves survival of ventral mesencephalic grafts to the 6-hydroxydopamine lesioned striatum. Exp Brain Res 1997, 116(1): 29-38.

31. Eslamboli AB, Georgievska RM, Ridley HF, Baker N, Muzyczka C, Burger RJ, Mandel L, Annett, Kirik D: Continuous Low-Level Glial Cell Line-Derived Neurotrophic Factor Delivery Using Recombinant Adeno-Associated Viral Vectors Provides Neuroprotection and Induces Behavioral Recovery in a Primate Model of Parkinson's Disease. The Journal of Neuroscience 2005, 25(4): 769.

32. Tadahiro Etoh YPK, Masahiko H, Michiko S, Shiming L, Chi-Tang H, Kanki K: Inhibitory effect of a formulated extract from multiple citrus peels on LPS-induced inflammation in RAW 246.7 macrophages. Functional Foods in Health and Disease 2013 3(6): 242-25.

33. Choi HD, Kang HE, Yang SH, Lee MG, Shin WG: Pharmacokinetics and first-pass metabolism of astaxanthin in rats. Br J Nutr 2011, 105(2): 220-7.

34. Grimmig B, Kim SH, Nash K, Bickford PC, Douglas SR: Neuroprotective mechanisms of astaxanthin: a potential therapeutic role in preserving cognitive function in age and neurodegeneration. GeroScience 2017, 39(1): 19-32.

35. Ji X, Peng D, Zhang Y, Zhang J, Wang Y, Gao Y, Lu N, Tang P: Astaxanthin improves cognitive performance in mice following mild traumatic brain injury. Brain Research 2017, 1659: 88-95.

36. Zhou XF, Zhang X, Hu J, Chen X, Wen Y, Sun Y, Liu R, Tang K, Zheng, Song Y: Inhibition of inflammation by astaxanthin alleviates cognition deficits in diabetic mice. Physiol Behav 2015. 151: 412-20.

37. Morganti JM, Nash KR, Grimmig BA, Ranjit S, Small B, Bickford PC, Gemma C: The soluble isoform of CX3CL1 is necessary for neuroprotection in a mouse model of Parkinson's disease. J Neurosci 2012. 32(42): 14592-601.

38. Zhao Y, Zhang Q, Xi J, Xiao B, Li Y, Ma C: Neuroprotective effect of fasudil on inflammation through PI3K/Akt and Wnt/beta-catenin dependent pathways in a mice model of Parkinson's disease. Int J Clin Exp Pathol 2015, 8(3): 2354-64. 
39. Chinta SJ, Andersen JK: Reversible inhibition of mitochondrial complex I activity following chronic dopaminergic glutathione depletion in vitro: Implications for Parkinson's disease. Free Radical Biology and Medicine 2006, 41(9): 1442-1448.

40. Kim GH, Kim JE, Rhie SJ, Yoon S: The Role of Oxidative Stress in Neurodegenerative Diseases. Exp Neurobiol 2015, 24(4): 325-40.

41. Mattei R, Polotow TG, Vardaris CV, Guerra BA, Leite JR, Otton R, Barros MP: Astaxanthin limits fish oil-related oxidative insult in the anterior forebrain of Wistar rats: Putative anxiolytic effects? Pharmacology Biochemistry and Behavior 2011, 99(3): 349-355.

42. Kang JO, Kim SJ, Kim H: Effect of astaxanthin on the hepatotoxicity, lipid peroxidation and antioxidative enzymes in the liver of CCl4-treated rats. Methods Find Exp Clin Pharmacol 2001, 23(2): 79-84.

43. Yurek DM, Fletcher-Turner A: Differential expression of GDNF, BDNF, and NT-3 in the aging nigrostriatal system following a neurotoxic lesion. Brain Research 2001, 891(1-2): 228-235. 